IV NEARBY ACTIVE GALAXIES AND THEIR NUCLEI

"Several years ago, before it was fashionable to feed the gas-eating monsters which are supposed to lurk in the nuclei of some galaxies ...."

R.J. Allen in Discussion IV.2 


\title{
RADIO PROPERTIES OF ACTIVE NEARBY SPIRAL GALAXIES
}

\author{
A. G. de Bruyn \\ Hale Observatories \\ Pasadena, California, USA
}

\section{INTRODUCTION}

Before reviewing the radio properties of active spiral galaxies it is appropriate, as well as instructive, to think about a working definition of an active galaxy. This may seem a trivial point when we consider classes of objects like Seyfert galaxies or radio galaxies but this is much less so when we inspect the nearby systems. In a sample of nearby galaxies one is likely to encounter much more milder forms of activity than that known from studies of more distant galaxies. This is, of course, due to the fact that the latter have been selectively taken from a much larger reservoir of objects. (Although this may seem a disadvantage from an observational point of view, there are some clear advantages as well. Firstly, we can obtain a much better linear resolution once we have been able to isolate the active nearby galaxies and, secondly, we eventually may hope to learn more about the physical situation that existed prior to the onset of the active period and how the surrounding medium reacts to the activity.)

Before proceeding then, first some comments about the qualitative observational definition of an active galaxy. Optically a galaxy is called active when its nucleus, which is a still poorly defined small volume in the central region, (1) emits significant amounts of non-stellar or non-thermal radiation, (2) contains excessive amounts of ionized gas, or (3) exhibits gas motions at velocities much larger than those reasonably ascribable to circular rotation. In the radio domain non-thermal emission is the rule rather than the exception and the classification of a spiral galaxy as active on the basis of its radio emission alone, requires the knowledge of what constitutes a "normal" amount of radio emission. The answer to that question is intimately related to the origin of relativistic particles in spiral galaxies and its variation with galactocentric distance. Since our knowledge of that subject is, at present, in a quite unsatisfactory state we have to employ a mostly empirical approach to the question of the radio continuum activity in 
spiral galaxies.

High-resolution one- and two-dimensional studies of spiral galaxies have indicated that the radio emission is generally dominated by a fairly axisymmetric disk component whose surface brightness increases towards the centre. (For a review see van der Kruit and Allen, 1976.) The disk-averaged brightness temperature at a wavelength of $21 \mathrm{~cm}$ lies in the range from 0.1 to 10 K (van der Kruit, 1973; Ekers, 1974b).

In Section 4 I will discuss several galaxies with large-scale disk structures with brightness temperatures considerably in excess of 10K. These structures are probably causally related to past nuclear activity although the nuclei do not necessarily exhibit any activity at present. The radio properties of presently optically active spiral galaxies will be discussed in Section 3 . First, however, I will consider the radio data on the central regions of nearby galaxies to assess whether they contain indications of ongoing nuclear activity.

\section{THE RADIO PROPERTIES OF THE CENTRAL REGIONS OF SPIRALS}

Recently Crane (1977) has completed an extensive survey of spiral galaxies with the NRAO interferometer. For an optically defined sample of 181 galaxies he has collected complete data about the total radio emission and that of central sources less than $1 \mathrm{kpc}$ in diameter and stronger than $20 \mathrm{mJy}$ at a wavelength of $11 \mathrm{~cm}$. In Table $1 \mathrm{I}$ have collected the properties of all those galaxies in his sample that satisfy the criterium that at least $25 \%$ of their radio emission originates in a region of less than 200 pc diameter. With this somewhat arbitrary criterium I do not claim to have separated the galaxies with active from those with quiescent nuclei but I think that this group certainly gives a fair representation of the radio and optical properties of spiral galaxies with possibly active radio nuclei. Note that the above-mentioned selection criterium implies that the brightness temperature of these central sources is at least one thousand times higher than that of the surrounding disks. Clearly some extra-ordinary processes must be going on. For comparison I also have listed the properties of the ultra-compact radio nuclei of M 82 and the Galaxy.

Although the number of objects in Table 1 is rather small I wish to draw attention to a few interesting correlations:

1) Active radio nuclei are found among all optical morphological types but there may be a tendency to avoid late-type spirals.

2) Only one galaxy in Table 1 has Seyfert characteristics (NGC 1068) and only one other (NGC 3504) emits intense optical emission lines from its 
Table 1: Galaxies with strong radio nuclei

\begin{tabular}{|c|c|c|c|c|c|c|c|c|}
\hline \multicolumn{2}{|c|}{ Galaxy } & \multirow{3}{*}{$\begin{array}{l}\text { Dist. } \\
\text { (Mpc) }\end{array}$} & \multicolumn{2}{|c|}{ Radio Size } & \multirow[t]{3}{*}{$\alpha^{a}$} & \multirow{3}{*}{$\mathrm{P}_{11 \mathrm{~cm}}^{\mathrm{b}}$} & \multirow[t]{3}{*}{$\%^{c}$} & \multirow[t]{3}{*}{ Ref. } \\
\hline & & & Angular & Linear & & & & \\
\hline NGC\# & Type & & $\left({ }^{\prime \prime}\right)$ & ( $\mathrm{pc})$ & & & & \\
\hline 1068 & $\mathrm{Sb}$ & 23 & $<0.5$ & $<56$ & -0.5 & 472 & 30 & 1 \\
\hline 2655 & SOp & 32 & $<1$ & $<155$ & -0.8 & 64 & $>65$ & 1 \\
\hline 3031 & $\mathrm{Sb}$ & 3.25 & $<0.0004$ & $<0.006$ & +0.2 & 0.8 & 25 & 2,3 \\
\hline 3079 & $\mathrm{Sc}$ & 24 & $<1.3$ & $<151$ & -0.4 & 56 & 30 & 4,5 \\
\hline 3504 & $S(B) b$ & 30 & $<0.2$ & $<29$ & -0.7 & 86 & 30 & 6,7 \\
\hline 4579 & $S(B) b$ & 19.5 & $<1$ & $<95$ & -0.3 & 13 & 50 & 1 \\
\hline 4594 & $\mathrm{Sa}$ & 19.5 & $\leq 0.0002$ & 0.02 & +0.3 & 36 & 100 & 2,8 \\
\hline 3034 & $\operatorname{Irr}$ & 3.25 & $\sim 0.0014$ & $\sim 0.022$ & -1.0 & $-\overline{3.3}$ & ----- & $\overline{9}, \overline{10}$ \\
\hline Sgr A & $\mathbf{S}$ & 0.01 & $\sim 0.017$ & 0.0008 & 0 & 0.0001 & 0.0005 & 11,12 \\
\hline
\end{tabular}

Notes: Spectral index defined as $\mathbf{S} \propto \nu^{\alpha}$

${ }^{b}$ In units of $10^{19} \mathrm{~W} \mathrm{~Hz}^{-1}$ Ster $^{-1}$

${ }^{c}$ Fraction of galaxy emission coming from central region

References: 1. Crane (1977); 2. de Bruyn et al. (1976); 3. Kellermann et al. (1976); 4. de Bruyn (1977b); 5. Seaquist, private communication; 6. de Bruyn and Wilson (1976); 7. Crane, private communication; 8. Kellermann, private communication; 9. Kronberg and Wilkinson (1975); 10. Geldzahler et al. (1977); 11. Lo et al. (1975); 12. Kellermann et al. (1977).

central region (Burbidge and Burbidge, 1962). Optical and radio activity therefore do not necessarily go together.

3) Although several galaxies in Table 1 have bright radio structures in the inner few kpc they do not have exceptional disk emission. This suggests that galactic nuclei do not contribute significantly to the cosmic ray reservoir in spiral galaxy disks.

Table 1 is nearly complete for galaxies with central sources having a luminosity greater than $10^{20} \mathrm{~W} \mathrm{~Hz}^{-1} \mathrm{Ster}^{-1}$ (since $90 \%$ of the galaxies in Crane's sample have a distance less than $25 \mathrm{Mpc}$ ). Of course, many more galaxies may exist that radiate more than $25 \%$ of their radio emission from a region less than $200 \mathrm{pc}$ in diameter, but these then must have both intrinsically faint nuclei and disks.

The radio nuclei of NGC 3031 and NGC 4594 stand out in Table 1 because of their inverted spectra. These are shown in Figure 1. Their exceptional 
a)

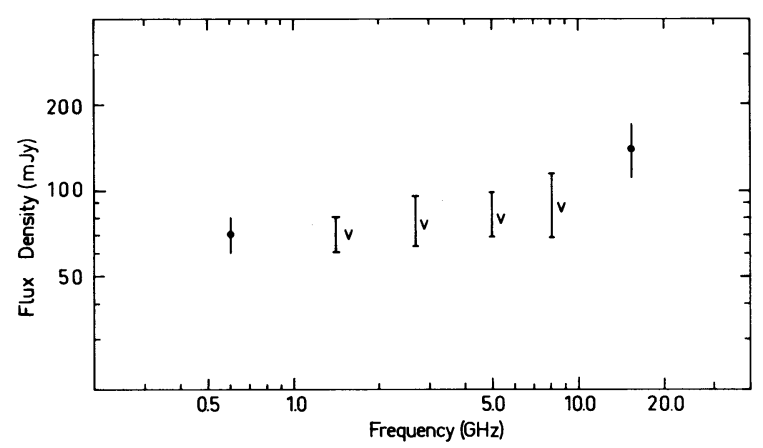

b)

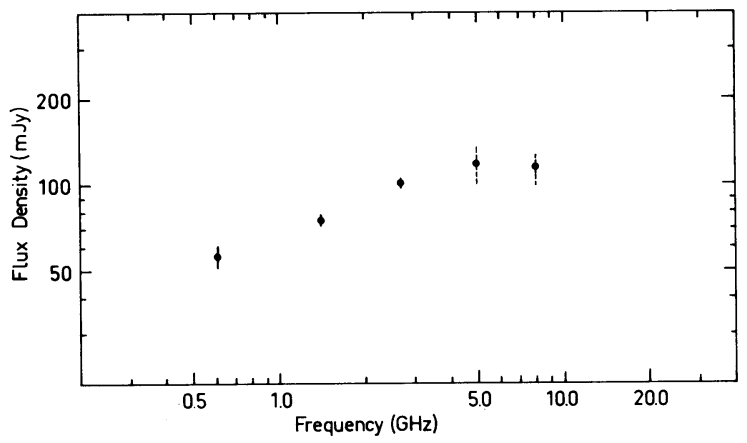

Fig. 1. a) Radio spectrum of the nucleus of NGC 3031 . The vertical bars at frequencies from 1.4 to $8.1 \mathrm{GHz}$ indicate the range over which the flux density varies. b) Radio spectrum of the nucleus of NGC 4594 (from de Bruyn et al., 1976).

character has been pointed out already several years ago by Ekers (1974a). Since the radio nuclei of these galaxies are also among the best studied we will now discuss their properties in more detail. The inverted spectra are most likely interpreted as due to self-absorption in non-uniform synchrotron sources with linear dimensions of a few times $10^{-3}$ pc (de Bruyn et al. 1976). An important property of these sources is their truly isolated nature with no significant emission coming from scales ranging upwards to several $\mathrm{kpc}_{\text {。 The }}$ source in NGC 3031 has been observed for nearly ten years now (the earliest data are from Wade (1968)), and this combined with the VLBI size limit indicates that the source cannot undergo an overall expansion at velocities exceeding about $500 \mathrm{~km} / \mathrm{s}$. This would appear to rule out an interpretation as a single supernova remnant and suggests a fixed source structure。 The same conclusion probably applies to the radio nuclei of NGC 4594 and M 82 (Kronberg and Wilkinson, 1975; Geldzahler et al., 1977). 


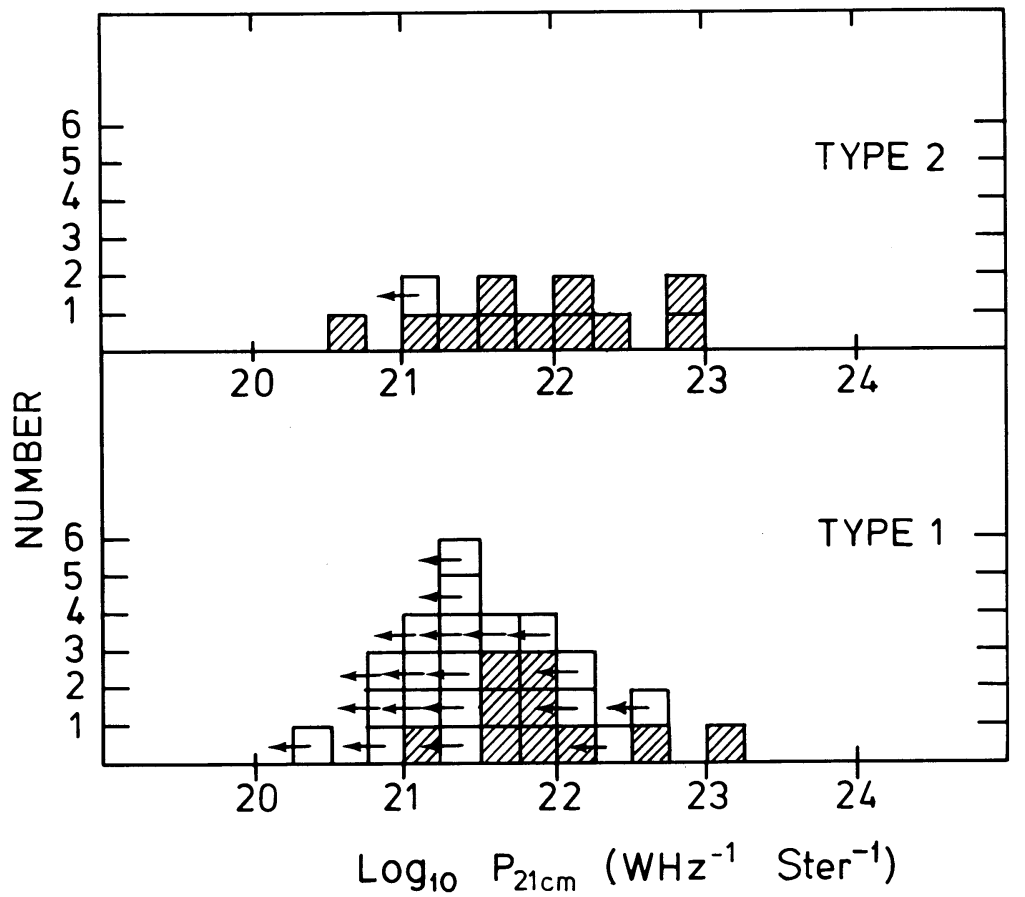

Fig. 2. Histograms of the luminosity distribution at $21 \mathrm{~cm}$ continuum of 12 type 2 and 29 type 1 Seyfert galaxies. Open boxes with arrows indicate upper limits for undetected galaxies (from de Bruyn and Wilson, 1978).

\section{RADIO EMISSION FROM OPTICALLY ACTIVE SPIRAL GALAXIES}

A surprising property of the nuclei of the galaxies described in the preceding section is that they exhibit little or no associated optical activity. In this section we will look at this problem from another way and investigate the radio properties of galaxies that are known or thought to be active on the basis of their optical properties.

\section{1 Sersic galaxies}

A group of galaxies that has been known for some time to possess peculiar optical nuclei are the so-called hot-spot nucleus galaxies investigated by Sersic and Pastoriza (1965) and Sersic (1973). Lequeux (1971) found a positive correlation between the presence of this type of nucleus and a radio nucleus. The study by Crane (1977) however, which involved a much larger group of Sersic galaxies, indicates no statistically significant correlation between the Sersic galaxies and radio emission from either disk or central 
region. The radio data therefore do not support a picture in which the hot spots would be the result of nuclear activity.

\subsection{Seyfert galaxies}

From the work of Wade (1968), Lequeux (1971) and van der Kruit (1971) it is known that the classical Seyferts have, on average, stronger nuclear radio sources than normal spiral galaxies. This result has recently been substantiated and extended to include also the tens of Seyfert galaxies discovered in Markarian's surveys (Sramek and Tovmassian, 1975; Sulentic, 1976; de Bruyn and Wilson, 1976). Figure 2 shows the distribution of radio luminosity of all the Seyfert galaxies in the first four lists of Markarian. Type 2 Seyferts are, on average, stronger radio emitters than type 1 Seyferts, a surprising result in view of the fact that the latter have stronger non-thermal optical continua. The Seyfert galaxies, both types taken together, occupy the upper end of the distribution of radio powers of normal spirals and frequently have luminosities exceeding by a factor of ten those of the most luminous normal spirals (Crane, 1977; de Bruyn and Wilson, 1978).

The sizes of the radio sources associated with Seyfert galaxies span the range from less than 1 parsec to more than $100 \mathrm{kpc}$ although the majority have a size of a few tenths of a kpc to several kpc (de Bruyn and Wilson, 1978). The apparent scarcity of ultra-compact opaque radio sources -- of the type seen in NGC 3031 and NGC 4594 -- need not be intrinsic since it may be due to the effects of free-free absorption by the large amounts of ionized gas known to exist in Seyfert nuclei.

An interesting but puzzling result is that none of the optically discovered Seyfert galaxies shows the double structure characteristic of so many other energetic extra-galactic radio sources (see also Section 4).

\section{LARGE-SCALE RADIO DISTURBANCES IN SPIRAL DISKS}

Ever since the discovery of double radio sources associated with elliptical galaxies radio astronomers have searched for similar phenomena around spiral galaxies. The original studies by de Jong (1965, 1966) and Tovmassian (1968) apparently indicated an excess of so-called satellite radio sources around spiral galaxies. More recent studies (summarized by Willis, 1976), however, failed to confirm these early results. I think it is true to say that no single case is known as yet where from the structure and/or alignment of such satellite sources a link with the central galaxy has been convincingly demonstrated.

This absence of double radio sources is now also found to extend to the 


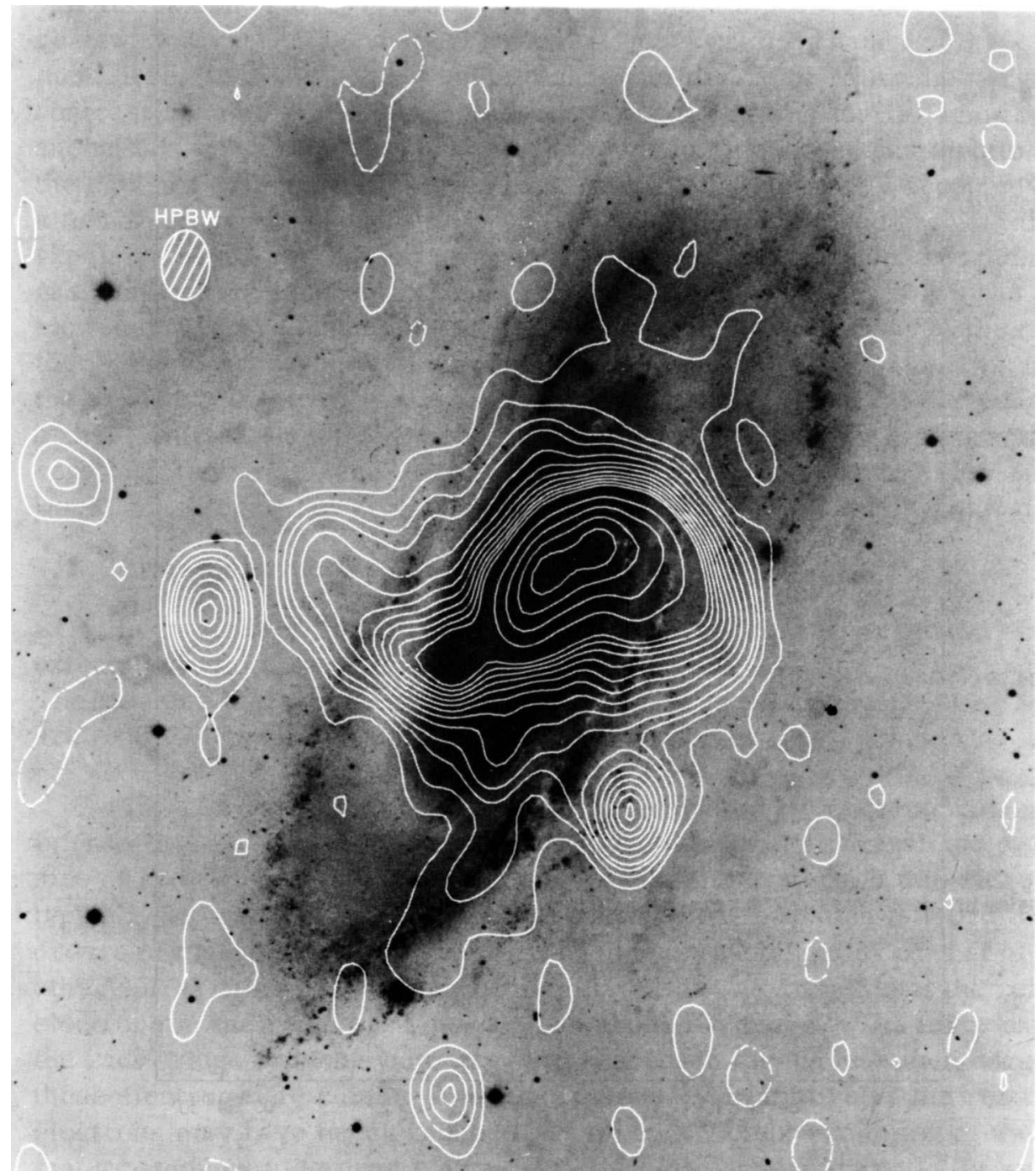

Fig. 3. Contour map of the $49 \mathrm{~cm}$ radio emission of NGC 4258 superimposed on an optical photograph (de Bruyn, 1977b). Contours are in units of $2.25 \mathrm{~K}$ brightness temperature up till $9 \mathrm{~K}$, then $4.5 \mathrm{~K}$ till $45 \mathrm{~K}$ and $22.5 \mathrm{~K}$ till $135 \mathrm{~K}$.

Seyfert galaxies, many of which may be classified as spiral galaxies. Most Seyferts are too distant to investigate with enough linear resolution whether this double structure in the radio remains absent when we get closer in to the seat of activity. We know that Seyfert galaxies eject large amounts of gas at velocities large enough to allow it to escape from the central region and one wonders what the fate of this gas is and how it interacts with the surrounding 


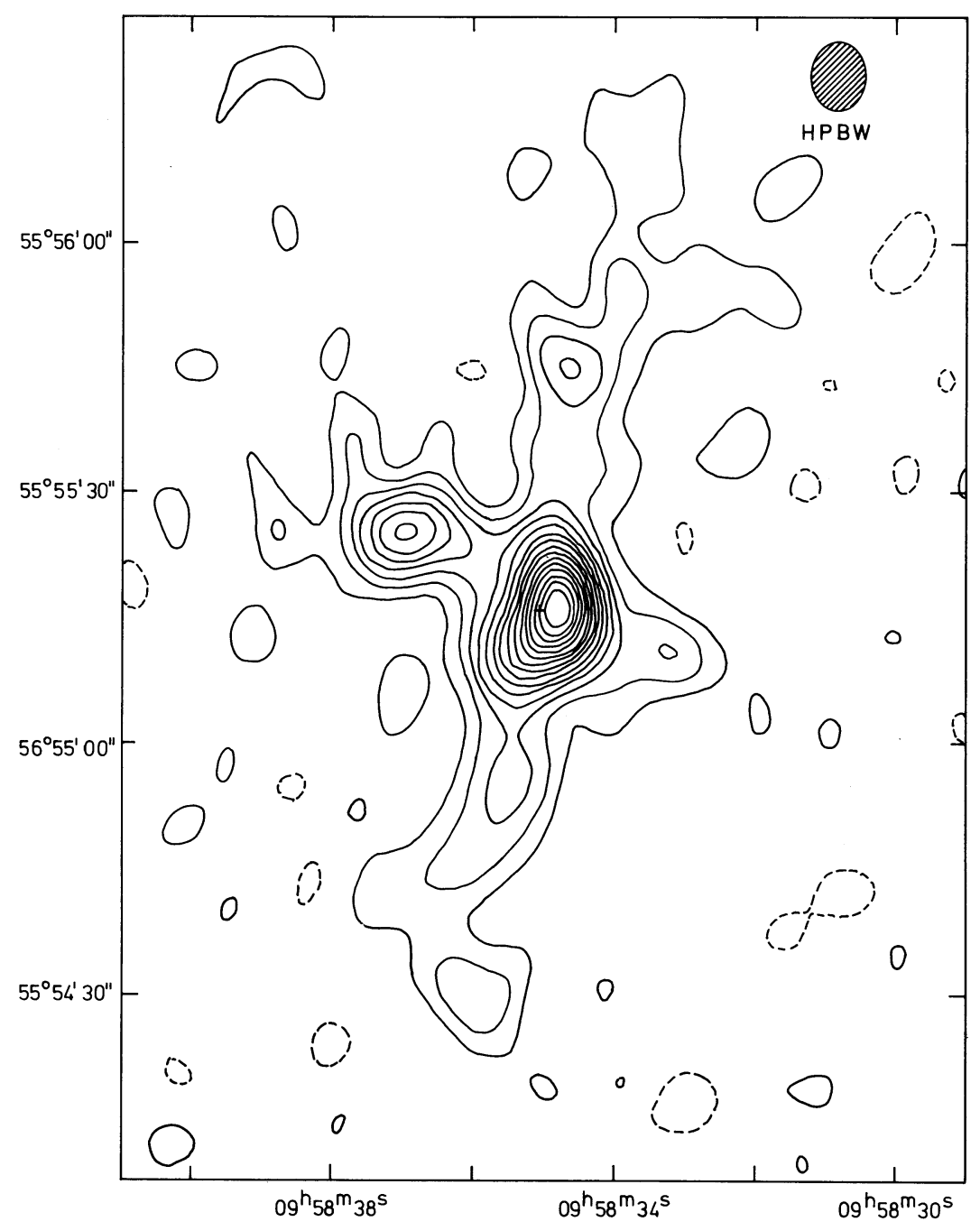

Fig. 4. Contours of the $6 \mathrm{~cm}$ radio emission of NGC 3079 (de Bruyn, 1977b). The contour interval is $1.5 \mathrm{~K}$ brightness temperature.

disks. With these questions in mind I will now present some high-resolution radio continuum maps of spiral galaxies whose radio morphology does not fit into the "standard" picture. Most of these results are from recent observations by the author with the Westerbork radio telescope (de Bruyn, 1977b).

\section{1 The case of NGC 4258}

This galaxy has been the subject of extensive investigations ever since the paper by van der Kruit et al. (1972) was published (van der Kruit, 1974; 
van Albada and Shane, 1975; de Bruyn, 1977b). Its properties have been reviewed at various occasions by Oort (e.g. Oort, 1974) and I will not repeat them here. A radio contour map is shown in Figure 3. Note the very large contrast in radio surface brightness between the normal spiral arms and the anomalous arms which are thought to be due to high-speed gas ejection from the nucleus. Although the latter explanation is not generally accepted no alternative theories have been put forward. The main difficulty with the expulsion hypothesis is perhaps the fact that no direct evidence of the ejected gas has been found although it is very unlikely that all ejected gas has been slowed down to velocities less than $50-100 \mathrm{~km} / \mathrm{s}$. Inspection of the map of total neutral hydrogen given by van Albada and Shane (1975) indicates that of the order of $10^{8} \mathrm{M}_{\odot}$ of gas is "missing" in the outer H I disk near the anomalous arms. This gas may have been swept out of the galaxy or shock-ionized to very high temperatures. Its detection by means of optical or X-ray emission would constitute a powerful argument in favor of the ejection hypothesis.

\subsection{The radio structure of NGC 2146 and NGC 3079}

In an attempt to find more cases like NGC 4258 I have recently made extensive radio observations of about ten galaxies with strong radio emission. The galaxies that come closest to NGC 4258 in terms of their radio properties are NGC 2146 and NGC 3079 which I will discuss in turn below.

The radio emission of NGC 2146 is dominated by a narrow ridge of intense emission centered on the nucleus and extending several kpc to either side of it (de Bruyn, 1977b). The brightness temperature in this ridge is typically of the order of $1000 \mathrm{~K}$ at $21 \mathrm{~cm}$ wavelength, which is more than three orders of magnitude higher than that of the surrounding disk or that of most other spiral galaxy disks for that matter. It can be argued that the relativistic electrons in the ridge are younger than about $10^{\circ}$ years. The large extent of the radio ridge and its symmetry with respect to the nucleus then suggest that these electrons are coming from the nucleus. Alternatively, the relativistic electrons may have been produced in-situ by a violent excitation of the interstellar medium due to gas ejection from the nucleus. It is probably relevant that the galaxy also looks very disturbed optically. Recently neutral hydrogen has been detected at distances up to $100 \mathrm{kpc}$ from the centre (Fisher and Tully, 1976), but the relation between this gas and the optical and radio continuum disturbances is unclear.

NGC 3079 has probably the most peculiar radio structure of any spiral galaxy studied thusfar. A radio contour map is shown in Figure 4. In addition to a bright radio nucleus (compare Table 1 ) it shows radio emission structured in the form of a cross (de Bruyn, 1977b; Seaquist et al., 1978). The arms of this cross in position angles $80^{\circ}-260^{\circ}$ are directed along the minor axis and reach to the projected edge of this nearly edge-on galaxy. The interpretation 


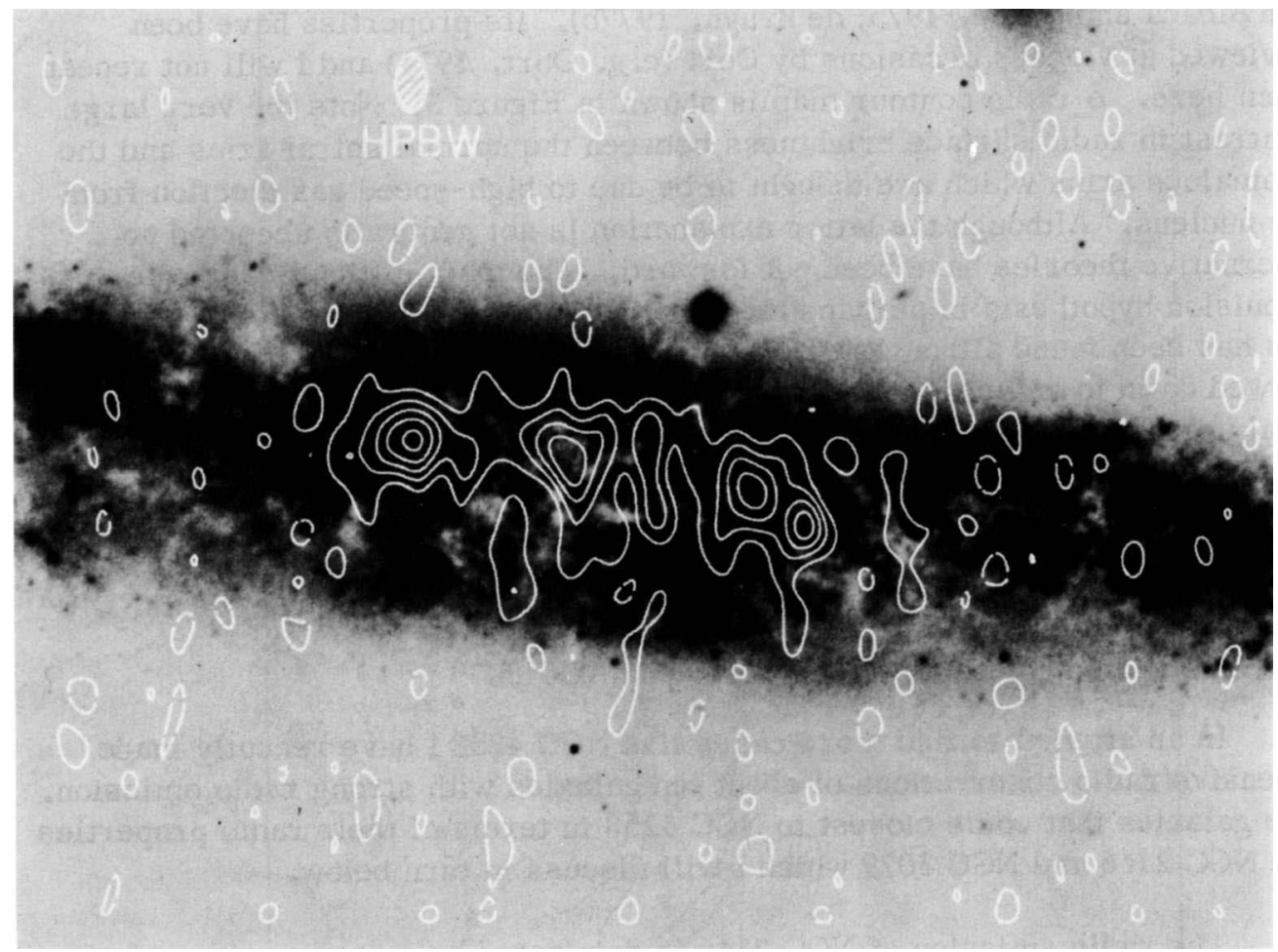

Fig. 5. Contour map of the $6 \mathrm{~cm}$ radio emission of NGC 4631 superimposed on an optical photograph (de Bruyn, 1977b). The contour interval is $1.0 \mathrm{~K}$.

of this phenomenon depends on whether the radio structure lies in the disk or is oriented perpendicular to the disk but in either case nuclear involvement seems likely. Also in this galaxy there are several optical features suggestive of nuclear activity.

\subsection{Other galaxies with peculiar disk radio features}

Among the tens of galaxies that have now been mapped with high spatial resolution NGC 4631 and NGC 4736 are noteworthy because of the presence of intense radio features in the central region that are fairly symmetrically located with respect to the nucleus (van der Kruit, 1971; de Bruyn, 1977a,b). A radio map of NGC 4631 is shown in Figure 5. NGC 5005 and NGC 5033 may also belong to this category although in these galaxies the double structures are more complex and their brightness enhancement over the base disk less dramatic (Seaquist, private communication). It is estimated that several percent of all spiral galaxies have radio components similar to those seen in these galaxies. 
Although the radio structures of these galaxies may be related to nuclear activity this interpretation is by no means unique and much more observational and theoretical work is needed. In particular it would be interesting to find out how tidal interactions between galaxies effect the relativistic component and magnetic fields in the disks of spiral galaxies.

Acknowledgements: I am indebted to Drs. P. C. Crane, K. I. Kellermann, E. R. Seaquist and G. D. van Albada for allowing me to use their data in advance of publication. This review was prepared while I held a Carnegie Fellowship and I am grateful to Dr. H. W. Babcock for his hospitality at the Hale Observatories.

\section{References:}

van Albada, G. D., Shane, W. W. 1975, Astron. Astrophys. 42, 433. de Bruyn, A. G. 1977a, Astron. Astrophys. 54, 491. de Bruyn, A. G. 1977b, Astron. Astrophys. 58, 221. de Bruyn, A. G., Crane, P. C., Price, R. M., Carlson, J. B. 1976, Astron. Astrophys. 46, 243. de Bruyn, A. G., Wilson, A. S. 1976, Astron. Astrophys. 53, 93. de Bruyn, A. G., Wilson, A. S. 1978, Astron. Astrophys. (in press). Burbidge, E. M.,Burbidge, G. R. 1962, Astrophys. J. 135, 694. Crane, P. C. 1977, The Radio Emission from Normal Spiral and Irregular Galaxies, Ph. D. Thesis, M.I.T.

Ekers, R. D. 1974a, in IAU Symposium No. 58 "Formation and Dynamics of Galaxies", Ed. J. Shakeshaft.

Ekers, R. D. 1974b, in "Structure and Evolution of Galaxies", Ed. G. Setti, D. Reidel Publ. Co., Dordrecht.

Fisher, J. R., Tully, R. B. 1976, Astron. Astrophys. 53, 397. Geldzahler, B. J., Kellermann, K. I., Shaffer, D. B., Clark, B. G. 1977, Astrophys. J (Letters) 215, L5.

de Jong, M. L. 1965, Astrophys. J. 142, 1336. de Jong, M. L. 1966, Astrophys. J. 144, 555. Kellermann, K. I., Shaffer, D. B., Pauliny-Toth, I. I. K., Preuss, E. Witzel, A. 1976, Astrophys, J. (Letters) 210, L121. Kellermann, K. I. Shaffer, D. B., Clark, B. G., Geldzahler, B. J. 1977, Astrophys, J. (Letters) 214, L61.

Kronberg, P. P., Wilkinson, P. N. 1975, Astrophys. J. 200, 430. van der Kruit, P. C. 1971, Astron. Astrophys. 15, 110. van der Kruit, P. C. 1973, Astron. Astrophys. 29, 263. van der Kruit, P. C. 1974, Astrophys. J. 192, 1. van der Kruit, P. C., Oort, J. H., Mathewson, D. S. 1972, Astron. Astrophys. 21, 169. van der Kruit, P. C., Allen, R. J. 1976, Annual Review Astron. Astrophys. Vol. 14, 417. 
Lequeux, J. 1971, Astron. Astrophys. 15, 30.

Oort, J. H. 1974, in IAU Symposium No. 58, "The Formation and Dynamics of Galaxies", Ed. J. Shakeshaft.

Seaquist, E. R., Davis, L., Bignell, R. C. 1977, preprint.

Sersic, J。 L. 1973, Publ. Astron. Soc. Pacific 85, 103.

Sersic, Jo L., Pastoriza, M. 1965, Publ. Astron. Soc. Pacific 77, 287.

Sramek, R. A., Tovmassian, H. M。 1975, Astrophys。 J. 196, 339.

Tovmassian, H. M. 1968, Astrophysics 4, 32。

Wade, C. M. 1968, Astron。 J. 73, 876.

Willis, A. G. 1976, Astron. Astrophys. 52, 219.

\section{DISCUSSION FOLLOWING REVIEW IV.1 GIVEN BY A.G. DE BRUYN}

ALLEN: The total radio flux density spectra of galaxies which are common1y called "active" very often have quite horizontal or even upturned and lumpy shapes at short centimeter wavelengths. I do not see how you find evidence for activity from the very straight spectra like $\mathrm{S} \propto v^{-0.7}$ which you have shown us for the last few galaxies.

DE BRUYN: The very fact that the spectra don't curve at high frequencies means that the electrons do not loose radiation to synchrotron and inverse Compton processes. The absence of a break in the spectrum indicates that the sources of the electrons must be very young, $\sim 10^{6}$ years; combined with the large linear extent and the high surface brightness of these galaxies this is an argument for activity.

BURBIDGE: How dependent is this result on the equipartition assumption?

DE BRUYN: Not very dependent, because the inverse Compton losses are of the same order as the energy in the stellar photons. I have taken a magnetic field strength of $10^{-5}$ Gauss which is equal to the equivalent field strength provided by the stellar photons. If the field strength is larger, the age limits become smaller than $10^{6}$ years.

EKERS: Could one not apply the same argument, i.e. the lack of a high frequency break in the radio spectrum, to the disk emission in which case we would call the whole disk "active"?

DE BRUYN: The spectra of the disks of quiet spirals, as I would call them, are not known at high frequencies. Also, the magnetic field strength and the inverse Compton losses are less in these galaxies, so the age 1imit for NGC 891 for example is at least a factor of 5 higher. This, combined with the fact that quiet spirals are brighter, would make them very exceptiona1.

VELUSAMY: The straight spectra shown for the nuclear emission of active galaxies are similar to those of the disk emission of spirals. Models for the disk suggest continuous injection and a leakage time of $<10^{7}$ years. Similarly, the nuclear sources with continuous activity 
could be much older than you estimated.

DE BRUYN: If the electrons would stay in the active galaxies $10^{7}$ years and would not be reaccelerated, you would see a break in the spectrum at a frequency far below $15 \mathrm{GHz}$. This we don't see. Hence, the age limit for the electrons in the active galaxies is probably a factor of 10 less than that in the disks of spirals.

\section{PREUSS: THE NUCLEUS OF NGC 1275 AT 2.8 CM WAVELENGTH}

The nucleus of the Seyfert galaxy NGC 1275 (3C 84) is one of the strongest extragalactic radio sources at $\mathrm{cm}$ wavelengths. Between 1960 and 1975 its flux density increased by more than a factor of 5, and has since remained relatively constant. High-resolution interferometric observations made at $1.3,2,2.8$, and $6 \mathrm{~cm}$ show that there are three main centers of emission with an overall extent of 0.006 arc sec (3 pc), aligned rough1y along position angle -9 degrees. Figure 1 shows the structure of 3C 84 based on measurements made at $2.8 \mathrm{~cm}$ (Schilizzi et al. 1975, Ap.J. 201, 263; Pauliny-Toth et a1. 1976, Nature 259, 17; and unpublished) at 5 epochs between March 1972 and Ju1y 1976.

During this period any relative motion of the components has been with an angular velocity $<50$ micro arc sec per year. If the source has expanded from a common point the velocity of the components would have had to be greater by a factor $\gtrsim 10$ during at least part of this time. The present low velocity of separation implies either deceleration of the components, or independent activity in the components. Although there is no evidence for component motion, the size of the individual components appears to have increased at a rate $\sim 30000 \mathrm{~km} / \mathrm{s}$ with the peak brightness temperature (of the central component) decreasing from $4 \times 10^{11} \mathrm{~K}$ to $1 \times 10^{11} \mathrm{~K}$.

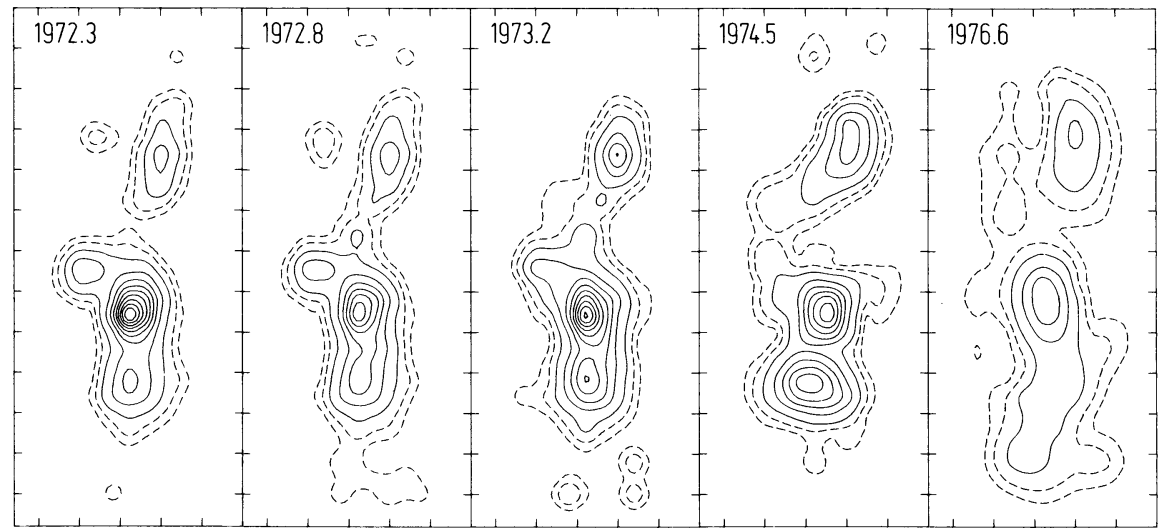

Figure 1. The structure of the radio nucleus of NGC 1275 as derived from VLBI data for five epochs. The spacing between tick marks is 0 ".001. The solid contours represent steps of $3 \times 10^{10} \mathrm{~K}$ brightness temperature, the dashed contours 0.5 and 0.25 of this value. 
EKERS: Cannot many of the triple and double sources in the central region of active galaxies be just as well described as somewhat irregular disks?

DE BRUYN: I agree that in some cases, 1ike NGC 4736 and 5033, the structure might be due to spiral arms. I haven't looked at optical photographs to check this. But I think NGC 1505 and 4631 have bright sources in the central regions.

SANCISI: No! The complex structure in the central region of the edgeon galaxy NGC 4631, which is found at $6 \mathrm{~cm}$, could be adequately explained by a $3 \mathrm{kpc}$ diameter edge-on disk. The two sources on opposite sides of the center, shown at $21-\mathrm{cm}$ resolution, could simply be due to spiral arms or ring structure of that small central disk.

DE BRUYN: We11, I disagree.

BURBIDGE: If you were given a sample of weak radio sources to map in this way, some associated with QSOs, some with radio galaxies, some with spirals, and some unidentified, could you from the maps identify the class of optical objects?

DE BRUYN: No, I could not. The only difference sometimes seen is a larger smooth background in the case of spirals due to the disk emission. But if you would take that smooth component away, some of these may look like extragalactic radio sources.

EKERS: Just for the record: I disagree.

VAN ALBADA: HI IN NGC 4258

NGC 4258 is an S(B)bc spiral galaxy with anomalous gaseous arms that have been observed in the radio continuum (van der Kruit et al. 1972, A.A. 21, 169) and in Ha (Courtès et a1. 1961, Compt. Rend. Acad. Sci. Paris 253, 218). These arms cut almost radially through the entire disk in diametrically opposed directions.

I have observed the galaxy in the $21-\mathrm{cm}$ 1ine of neutral atomic hydrogen. The observations were made with the Westerbork Synthesis Radio Telescope and with the 100-m dish of the Max-Planck-Institut für Radioastronomie in Bonn. The most important observed features are:

(1) In agreement with most theoretical predictions there is a distinct lack of HI emission in and in front of the anomalous arms. The emission temperature of the anomalous arms is low enough that absorption cannot be the main cause of this lack of emission.

(2) Outside $10 \mathrm{kpc}$ the disk of NGC 4258 shows nearly circular rotation, together with a small but distinct contraction along the minor axis.

On theoretical grounds it is impossible to explain this contraction as the aftereffect of an explosion in the nucleus.

(3) Inside $10 \mathrm{kpc}$ very large non-circular motions begin to occur. The contraction along the minor axis reaches velocities up to at least $100 \mathrm{~km} / \mathrm{s}$. A velocity jump of at least $200 \mathrm{~km} / \mathrm{s}$ occurs at a distance strongly reminiscent of the velocity field seen in theoretical computa- 
tions of gas response to a bar. The presence of a bar at the correct position angle is suggested by the total HI map and by some optical photographs. The morphological evidence is rather weak however. The velocity jump lies near the front (leading) edge of the anomalous arms, and could also be explained, at least in part, by the same explosive mechanism that is used to explain the anomalous arms.

BALDWIN: What criteria were used to define the major and minor axes in NGC 4258 ?

VAN ALBADA: The axes were determined from a best fit to the velocity field beyond $10 \mathrm{kpc}$ from the center. The deviation from the axes is about $10 \mathrm{~km} / \mathrm{s}$ for both the major and the minor axis, but the deviation from the minor axis is very consistent over a large distance.

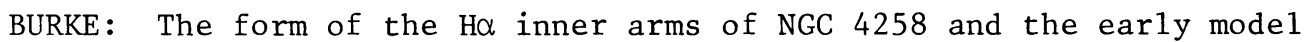
calculations gave strong support for nuclear ejection as a mechanism. It is surprising, though, that the overall velocity field of the HI shows so little perturbation. Rather than appealing to nuclear ejection, therefore, can the theorists find a catastrophic mode of spiral arm formation that produces the arms directly through the infalling material forming a shock or hydraulic jump with enough energy to ionize the medium? The general inflow along the minor axis might be evidence for material falling into the gravitational perturbation of the density wave.

SHU: Ten $\mathrm{km} / \mathrm{s}$ is much too small to ionize hydrogen completely in a shock. Velocities of order $50-100 \mathrm{~km} / \mathrm{s}$ are needed.

VAN DER KRUIT: Velocity differences in NGC 4258 are much larger than $10 \mathrm{~km} / \mathrm{s}$ and spectra show that the line strengths are consistent with collisional excitation.

DE BRUYN: The fact that we hardly ever see noncircular motions in HI exceeding a value of about $100 \mathrm{~km} / \mathrm{s}$ in the disks of spiral galaxies may be partly due to a "selection" effect. Namely, if gas would move at much higher velocities it will be collisionally ionized due to interaction with the interstellar medium, reach a very high temperature, and may expand from the disk before it could recombine to produce observable optical line emission or $21-\mathrm{cm}$ line radiation.

OORT: One should point out that in the inner parts of the anomalous synchrotron arms in NGC 4258 ionized hydrogen arms are observed which fit so closely to the synchrotron arms that they are undoubtedly connected with these. This shows that at least in this part of the synchrotron arms the gas has been ionized. It is plausible that further out the gas would have still higher temperatures and would therefore not be visible in HI. It is likely that what we do observe in HI is connected with subsidiary stages. 